\title{
Measurement of the isomeric yield ratios of fission products with JYFLTRAP
}

\author{
D. Gorelov ${ }^{1}$, A. Al-Adili ${ }^{3}$, J. Hakala ${ }^{1}$, A. Jokinen ${ }^{1}$, V. S. Kolhinen ${ }^{1}$, J. Koponen ${ }^{1}$, M. Lantz ${ }^{3}$, \\ A. Mattera ${ }^{3}$, I. Moore ${ }^{1}$, H. Penttilä ${ }^{1}$, I. Pohjalainen ${ }^{1}$, S. Pomp ${ }^{3}$, V. Rakopoulos ${ }^{3}$, M. Reponen ${ }^{1}$, \\ S. Rinta-Antilav ${ }^{1}$, V. Schonnenschein ${ }^{1}$, V. Simutkin ${ }^{3}$, A. Solders ${ }^{3}$, A. Voss ${ }^{1}$ and J. Äystö ${ }^{2}$ \\ ${ }^{1}$ University of Jyväskylä, Jyväskylä, Finland \\ ${ }^{2}$ Helsinki Institute of Physics, Helsinki, Finland \\ ${ }^{3}$ Uppsala University, Uppsala, Sweden
}

\begin{abstract}
Several isomeric yield ratios of fission products in $25 \mathrm{MeV}$ proton-induced fission of ${ }^{238} \mathrm{U}$ were measured recently at the JYFLTRAP facility. The ion-guide separator on-line method was utilized to produce radioactive ions. The double Penning-trap mass spectrometer was used to separate isomeric and ground states by their masses. To verify the new experimental technique $\gamma$-spectroscopy method was used to obtain the same isomeric ratios.
\end{abstract}

\section{Introduction}

Nuclei having the same charge and mass numbers but could be distinguished only by other properties, particularly radioactive ones are called isomeric nuclei. A nuclear isomerism happens due to structural effects. In terms of a shell model isomeric states exist when major shells occupied by particles of high angular momentum are preceded by closely lying subshells occupied by particles of low angular momentum [1]. In these cases electromagnetic transition probabilities are strongly reduced because the multipolarity of $\gamma$-quanta is quite high and emitted energy is relatively small.

As result, the isomeric states have a wide range of lifetimes. In Ref. [2] the range from $\sim 10^{-6}$ to $\sim 10^{10} \mathrm{~s}$ is mentioned. Thus, the population of isomeric states can dramatically change the time dependent description of some physical processes. For example, the time development of the decay energy release following binary fission depends on the initial relative populations between isomeric and ground states [2]. Thus, experimental data on the isomeric yield ratios are very important for decay heat calculations for reactors.

Different techniques exist to measure the independent isomeric yield ratios. They include isotope separator [3], recoil mass separator [4] or radiochemical methods in combination with $\gamma$-ray spectroscopy. The bottleneck all of this methods is the detection of isomeric and ground states by their decays. To estimate the initial ratio between population of the isomeric and the ground state, information about the decay scheme, the branching ratios etc. is required.

A complitely different method to measure isomeric yield ratios of fission products was proposed at the Accelerator Laboratory of the University of Jyväskylä [5]. The main idea is to combine the ion guide technique and the unique capabilities of JYFLTRAP [6] to separate the isomeric and the ground state $[7,8]$. In this method two states are distinguished due to the mass difference. Previous experiments have already demonstrated that the resolving power of the facility is enough to perform isomeric yield ratio measurements for several cases [7,9-11].

\section{Experimental method}

The experimental measurement of independent isomeric yield ratios of fission products recently has been carried out at JYFL. The isomeric yield ratios have been determined in $25 \mathrm{MeV}$ proton-induced fission of ${ }^{238} U$. Two different methods have been utilized in the experiment. First technique is based on isobaric 
separation of the fission products and detection of the isomeric and the ground state by their $\gamma$-decays. The main idea of the second method is to separate the isomeric and the ground state with JYFLTRAP and count ions with microchannel plate (MCP) detector.

The schematic view of the experimental facility is presented on the Fig. 1. The uranium target,

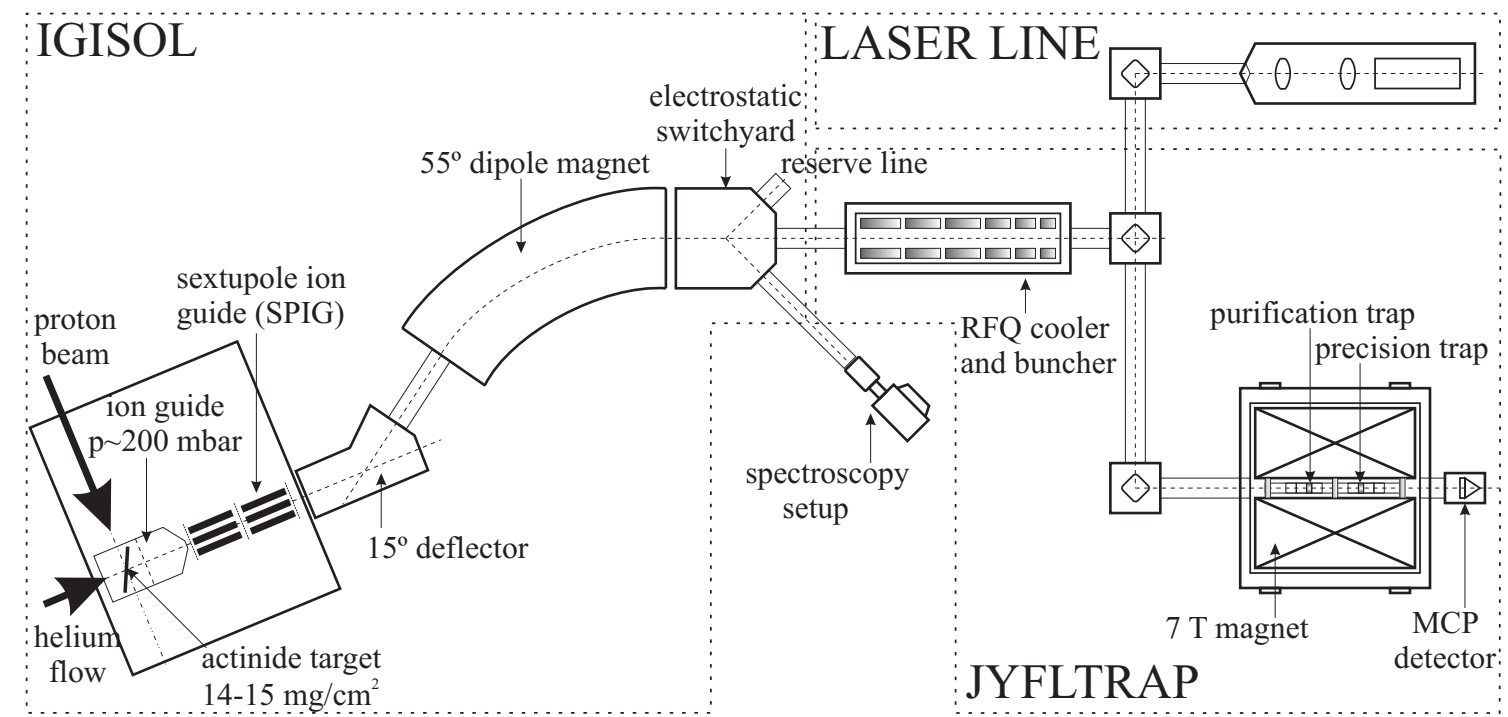

Fig. 1: A schematic view of the IGISOL-4 facility. Due to new layout it is possible to make various experiments employing different setups and techniques. The double Penning trap and $\gamma$-spectroscopy system were used in the present work.

$15 \mathrm{mg} / \mathrm{cm}^{2}$ thick, is placed in the fission ion-guide. Ions produced in nuclear fission are stopped in the ion-guide filled with helium gas at a pressure of around 200 mbar. Due to high ionization potential of the buffer gas, after slowing down a considerable fraction of ions aquires charge state +1 . The ions are extracted from the gas cell by differential pumping and transported futher with a sextupole ion-guide (SPIG) [12]. After an acceleration to $30 \mathrm{kV}$ the continuous ion beam is separated with a $55^{\circ}$ dipole magnet. As result, the isobaric chain with certain mass number $\mathrm{A}$ is selected.

The new construction of the IGISOL facility at JYFL [13] gives the opportunity to distribute separated beams between different setups. After the electrostatic switchyard the beam can be send towards JYFLTRAP or it can be directed to the $\gamma$-spectroscopy setup.

The Penning trap measurements require specially prepared beam. For this purpose ions are injected into a gas-filled radio-frequency quadrupole cooler and buncher (RFQ) [14]. The RFQ system cools ions and releases them as short bunches to JYFLTRAP.

JYFLTRAP $[6,16]$ consists of two cylindrical Penning traps inside a 7-T superconducting solenoid. The fact that there are two traps in one magnet is very important. It allows us to utilize two stage purification technique [17] and achive a mass resolving power about $m / \Delta m \approx 10^{6}$. However in the present experiment the isomeric and the ground states were separated by the purification trap with a buffer gas cooling technique $[15,18]$.

When ions are inside the trap, dipole excitation is applied to the electrods. As result, all ions regardsless at their mass are moved to a large radius. After that, applying a quadrupole radio-frequency field will center only ions whose cyclotron frequency $f_{c}(m)$ matches with applied frequency. The cyclotron frequency $f_{c}$ of an ion with a mass $m$ and a charge $q$ is given as:

$$
f_{c}=\frac{1}{2 \pi} \frac{q}{m} B
$$


where $B$ is the magnetic field.

Recentered ions are released from the trap through a $2 \mathrm{~mm}$ diameter diaphragm and registered by $\mathrm{MCP}$ detector. In the present measurements full cycle has been chosen 660 milliseconds. The final spectrum shows how many ions were detected for each quadrupole excitation frequency.

To test the new method and compare results, $\gamma$-spectroscopy mesurement was done in the same experiment. The constant beam after the dipole magnet (see Fig. 1) was directed to the spectroscopic station. Ions were implanted on the aluminium foil, which was placed in front of the detector. The single $\gamma$-spectrum was collected in several hours.

\section{Results}

In the present experiment isomeric yield ratios were measured for several cases utilizing the Penning trap technique and the $\gamma$-spectroscopy. Data analysis is still on going. That's why only preliminary data on ${ }^{81} \mathrm{Ge}$ are presented in this report.

On the Fig. 2 the quadrupole frequency spectrum is presented for Ge and As ions. Black circles are

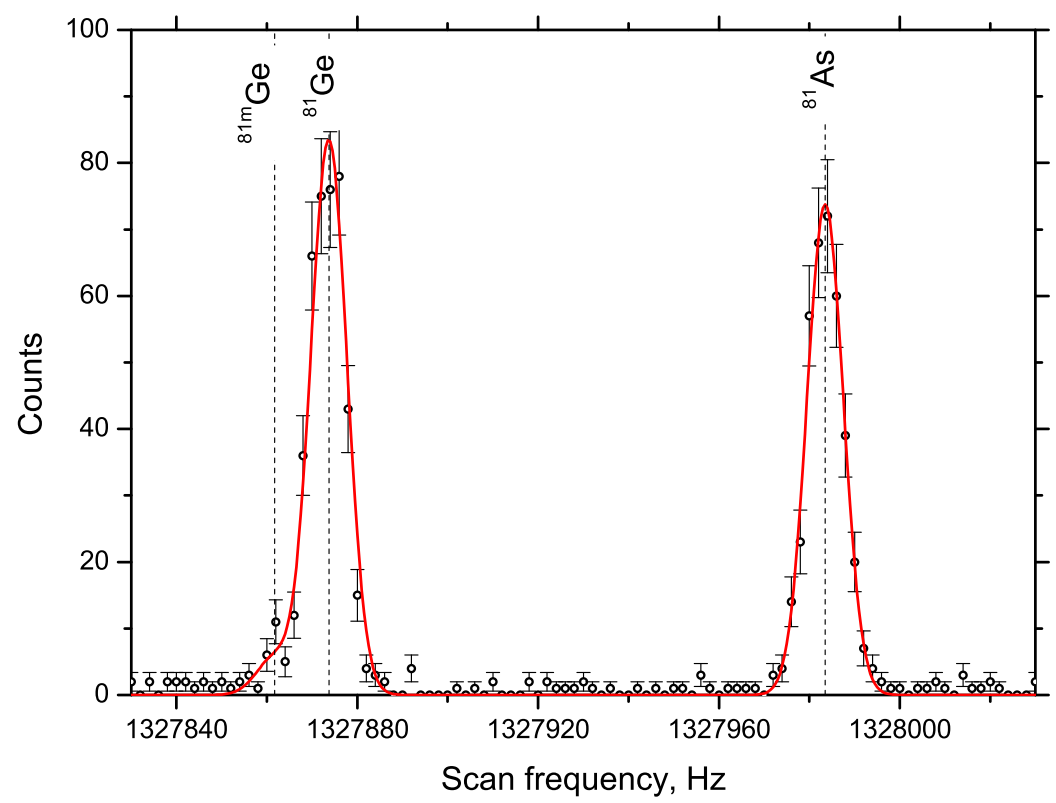

Fig. 2: The quadrupole frequency spectrum collected after the Penning trap. Black circles are experimental points with statistical error bars. Red line is gaussian fit of the spectrum. Position of peaks was calculated according to ion masses.

experimental points with statistical error bars. The difference between the isomeric and the ground state of ${ }^{81} \mathrm{Ge}$ is $679 \mathrm{keV}$. This value is very close to maximum resolving power of the purification trap. That's why the peak position for ${ }^{81 m} \mathrm{Ge}$ and ${ }^{81} \mathrm{Ge}$ were calculated according to their masses. The peak position of ${ }^{81} \mathrm{As}$ was taken as a reference. Peak width was chosen equal for all peaks. Using this assumptions experimental data were fitted by gaussian function (red line on the Fig. 2). The ratio between respective peak areas gives us the isomeric yield ratio for ${ }^{81} \mathrm{Ge}$. It is around 0.07 in the case of ${ }^{81} \mathrm{Ge}$. The uncertainty has not been estimated yet.

Another way to get the same parameter is to measure $\gamma$-decays of the isomeric and the ground state 
of ${ }^{81} \mathrm{Ge}$. Such measurement was done in the same experiment. A spectrum of $\gamma$-rays from isobaric chain with mass number $A=81$ is presented on the Fig. 3. It is more difficult to extract data from $\gamma$-spectrum

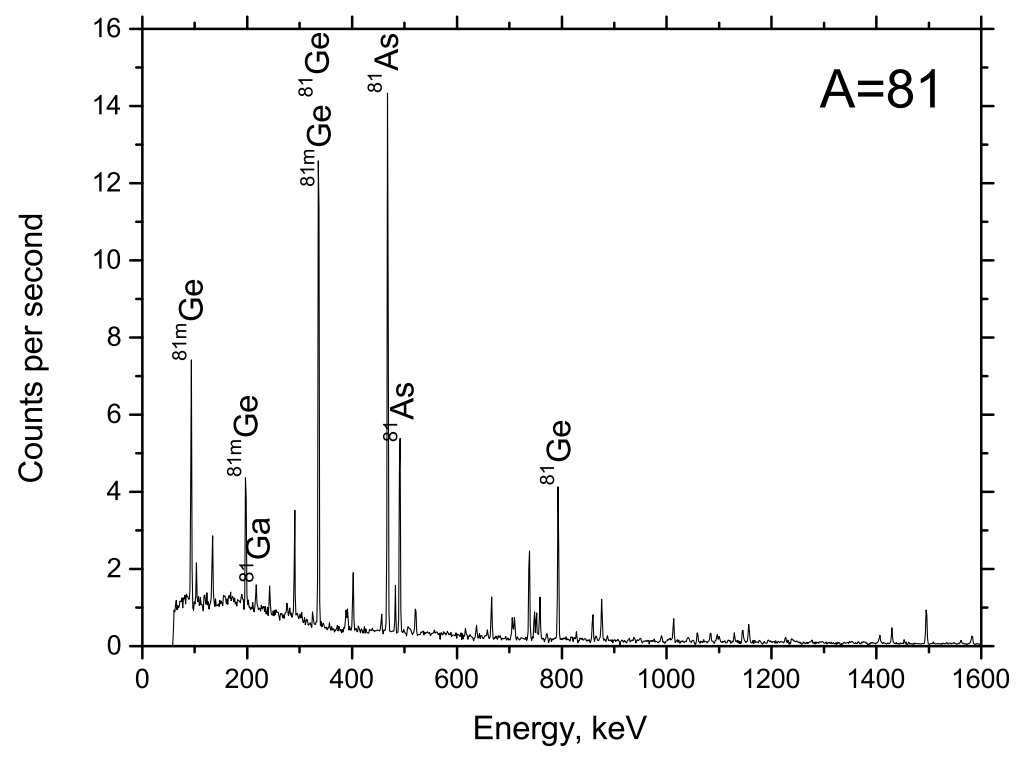

Fig. 3: Spectrum from the decay of all elements produced in $25 \mathrm{MeV}$ proton-induced fission of ${ }^{238} \mathrm{U}$ with mass number 81 . Laboratory background was subtracted from the spectrum. Several peaks related to the mass spectrum are identified.

than from data obtained with the Penning trap. In the case of $\gamma$-spectroscopy it is necessary to account for detector efficiency, branching ratio of decays, feeding decays of elements which have the same mass number etc.

Nevertheless, $\gamma$-spectroscopy was only the way to perform such measurements in the past. A very similar experiment utilizing the IGISOL method and $\gamma$-spectroscopy technique was done by M. Tanikawa et al. [3]. Data analysis is in progress and comparison between two different methods and literature values will be performed in future.

\section{Acknowledgements}

This work has been supported by the Academy of Finland under the Finnish Center of Excellence Programme 2012-2017 (Nuclear and Accelerator Based Physics Programme at JYFL) and by EU 7th framework programme "European Research Infrastructures for Nuclear Data Applications" under the project 269499. Authors also acknowledge the support from the Academy of Finland under the project 139382 (Precision fission studies for practical needs).

\section{References}

[1] E. Segre and A. C. Helmholz, Rev. of Mod. Phys. 21 (1949) 271.

[2] D. G. Madland and T. R. England, Nucl. Sci. and Eng. 64 (1977) 859.

[3] M. Tanikawa et al., Z. Phys. A 347 (1993) 53.

[4] F. Gönnenwein et al., Int. J. of Mod. Phys. E 16 (2007) 410.

[5] D. Gorelov et al., Proc. of ICFN5, Sanibel Island, USA, 2012 
[6] T. Eronen et al., Eur. Phys. J. A 48: 46 (2012).

[7] H. Penttilä et al., Eur. Phys. J. A 44 (2010) 147.

[8] H. Penttilä et al., Eur. Phys. J. A 48 (2012) 43.

[9] K. Peräjärvi et al., App. Rad. Isot. 68 (2010) 450.

[10] K. Peräjärvi et al., App. Rad. Isot. 71 (2013) 34.

[11] A. Kankainen et al., Phys. Rev. C. 87 (2013) 024307.

[12] P. Karvonen et al., Nucl. Instr. and Meth. B 266 (2008) 4794.

[13] I. D. Moore et al., Nucl. Instrum and Meth. B 317 (2013) 208.

[14] A. Nieminen et al., Nucl. Instr. and Meth. A 469 (2001) 244.

[15] V. S. Kolhinen et al., Nucl. Instr. and Meth. A 528 (2004) 776.

[16] V. S. Kolhinen et al., Nucl. Instr. and Meth. B 317 (2013) 506.

[17] T. Eronen et al., Nucl. Instr. and Meth. B 266 (2008) 4527.

[18] G. Savard et al., Physics Letters A 158 (1991) 247. 